\title{
SMART INTELLIGENCE FOR EFFICIENT HOME AUTOMATION SYSTEM
}

\author{
Ramya S \\ Assistant Professor, Dept of ISE, Maharaja Institute of Technology Mysore, India \\ Priyanka G, Rishika Rajagopal, Theertesh Y, Vishrutha M \\ Engineering Students, Dept of ISE, Maharaja Institute of Technology, Mysore, India
}

\begin{abstract}
In recent years, the Internet of Things (IOT) is getting more tractions. One of the real world examples of IOT is smart home. Smart home delivers home automation for installed devices at home such as lighting, air conditioning, temperature control etc. It permits the device which is connected to internet to be observed and managed at a distance by the user. Furthermore many researches on smart home concept concentrate only on some of the main feature. They still lack on the importance of using IOT i.e. providing energy efficiency, monitoring the energy usage, providing adequate security and protecting privacy. In this paper we propose a smart home automation system which consists of Raspberry pi and Node MCU to automate the system that serves as merely a switch replacement and it also records and reports important things which should be known by the owner of house. We fruitfully inspect our proposed system in a real world working condition. The interaction of the user and the system is made through Blynk application. Safety is an important measure in the house. In our paper we are focusing on two main important features firstly, designing a prototype of smart door lock system which can be used to intensify security of a door. Here we are making use of OTP based door locking technology at the door knob for automatic door lock purposes. Secondly, energy efficiency is provided by implementing an efficient system where energy consumption is monitored by recording the energy produced and consumed.
\end{abstract}

\section{INTRODUCTION}

The primary need of society is the shelter. With the increasing economic conditions, the demand of having a safe and comfortable life for people is also increasing. Technology is one of the things that are able to affect the security and can create comfort of a home [1]. The main objective of the smart home is that to make homeowners feel more comfortable and safe may be he is inside or outside the house. Smart homes were once acclaimed for the rich domain but gradually, it is becoming a requirement for many people in urban areas due to its ubiquitousness, as and when the price are going downwards year by year. IoT assists smart home in various aspects and among them that grabs the attention of the people are security and automation aspects.

Smart home is a new concept and it is growing day by day introducing new features. In 1995, a company in Scotland developed the first smart home technology which was named as X10. The computer system in a smart home can keep track of many aspects of daily life hence; a smart home takes shape as intelligence. One of the useful and advantageous features of a smart home is as the number of controllable devices increases; the ability to interconnect and communicate these devices digitally becomes easy. In the year 1999, Kevin Ashton was the first person who initiated the term IOT, where the device is not naturally connected to the internet, but has the ability to monitor and control them [2]. As we come across in some of the researches on smart home, they have given importance only to some of the aspects on how to attain energy efficiency, monitoring, security enhancement and preserving prvacy [3]. Therefore, to fill the voids in the IoT research, we have designed and buikt a smart home prototype using Raspberry $\mathrm{Pi}$ and Node MCU that combines the enhanced features. [4]. We proposed a smart home that not only focuseson the lighting aspect, i.e. substitutin mechanical switches to turn on or off electrical devices but it also records and reports some important issues to the home owner, such as home security. Our proposed work gives a report of the intruders. We can also calculate the power consumption of the electrical devices so the home owners can save energy which plays an important role in saving the environment. To a great extent, the home owner can keep an eye on their home when the person is in the home and also when the person is not at home. Also generation of electrical energy is done through solar panels which help in energy conservation and the control is automated [5]. The system is been designed such that it provides a graphical user interface i.e. which enables the user to control and monitor the system through the smart phone [6].

\section{PROBLEM STATEMENT}

In the latter day home automation is flattering indispensable for the need of improving our life circumstances. Smart home system furnishes favorable way of life in which everyone acquires to authorize his/her entire home using a smart phone, from turning on a light, TV to locking/unlocking doors, windows; it also furnishes a well organized use of energy. Automation provides controlling the energy resources according to the human need. When 


\section{International Journal of Engineering Applied Sciences and Technology, 2020 \\ Vol. 5, Issue 4, ISSN No. 2455-2143, Pages 657-662 \\ Published Online August 2020 in IJEAST (http://www.ijeast.com)}

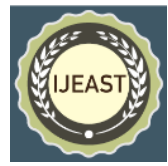

we don't require energy it automatically gets switched off and thus save the power.

To acquire such system installed in the home will be expensive and this is the main reason of why smart home has not received much order and awareness, and also the problem of installing and configuring it. Thus it is very important to make it reasonable and easy to configure, if this is done, people will be willing to implement it in their homes, companies and colleges. In other words, smart home requires moderation in order to lower the price of implementing it to the homes. Also smart home offers ease of use to the physically handicapped and also old age people in their homes by just one click to perform whatever they need.

\section{EXISTING SYSTEM}

As stated by the researches, the major problem in existent home automation system is their execution and preservation cost which cannot be afforded by most of the users. Moreover, some of the existing systems furnish a perspective of the house where application is provided to the users, i.e. inconvenient for them, in which they need to access the Web cam all the time when they have to see their houses. Few automation systems lack in maintaining and executing the home devices. And there are also some other constraints in the communication technologies that are in the existing automation systems. For example, Bluetooth range is limited to 10 meters, so this is the problem where user is not able to monitor his/her house device. Some technology like GSM can be accessed everywhere throughout the world but it is expensive and it has constraints in covering rural areas.

\section{PROPOSED SYSTEM}

We present a creative system to solve the constraints of existing smart home system. It can be attained by designing and fabricating a moderate $\mathrm{Wi}-\mathrm{Fi}$ based automotive mode for smart home model by using arduino microcontroller, Raspberry pi and android/ios based smart phone. The system is designed to control all the electrical devices at home through easy and efficient way. The framework is expanded using Raspberry pi and Node MCU as the major elements. Other elements used in the expansion of this model are sensors that consists of DHT11 (temperature and humidity) sensor, ACS712 current sensor and voltage sensor. Sensors will forward the data to Raspberry pi results, then the Raspberry pi will order relay to switch on/off the lamp, fan that are in the home. DHT11 sensor is used to indicate the surrounding temperature and humidity in the home.

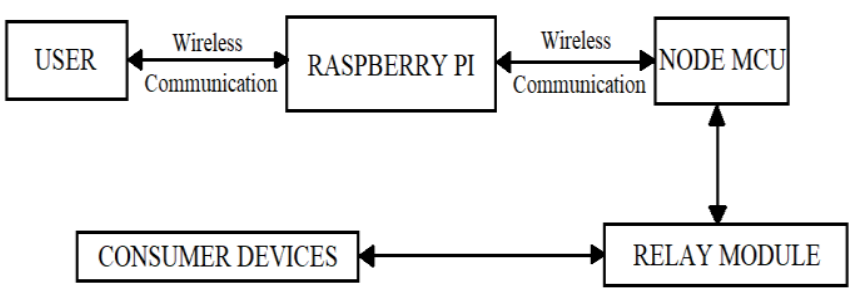

Fig1: Block diagram of the proposed model

\section{METHODOLOGY}

In our project we have implanted the ESP8266 Wi-Fi module with 4 channel relay to sway device beamed or from certain distance. In our project we utilize hotspot configuration to attain our project goal, first we create a hotspot channel to associate all the devices. And then we configure the correct IP address which the software generates i.e. "Arduino IDE" for all the devices to associate. IP will always be constant because the ESP module system is stable. Arduino IDE associates to the Arduino hardware to upload the program and impart with them.

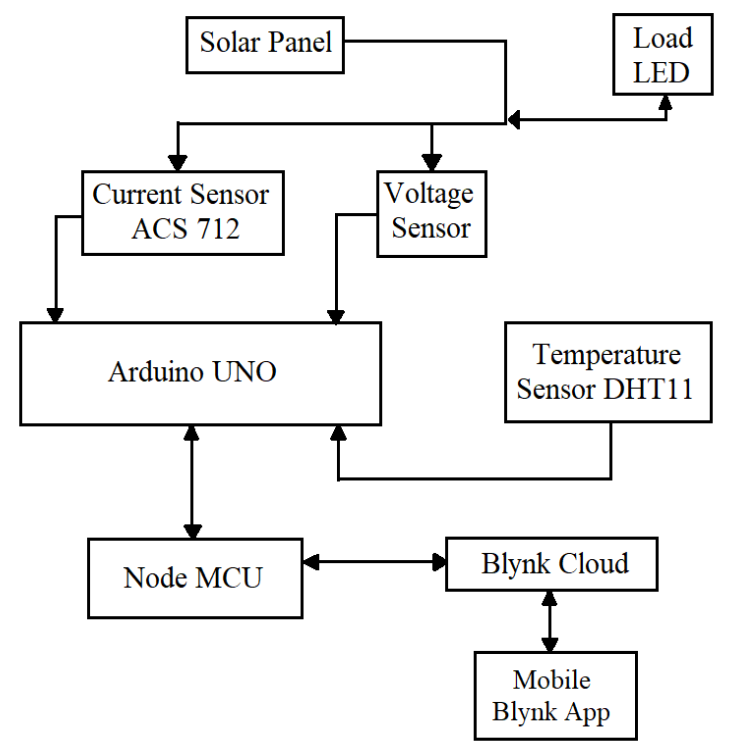

Fig2: System Architecture of Solar Monitoring System

In our project we concentrate on the utilization of Raspberry $\mathrm{Pi}$ and Wi-Fi module ESP8266 in Home Automation System for supervising all the devices at home. A suitable Wi-Fi based android application called Blynk App is used since it is comforting interface and it also works efficiently with aurduino controller to supervise the devices via smart phone. Arduino controller is programmed to interrelate with Blynk App. The solar panel, LED Load, temperature and humidity sensor DHT11, current sensor ACS712 and voltage sensor will be attached to Arduino and Node MCU. Sensors are constituted as input of the microcontroller. Both current and voltage sensors are utilized for keeping track of the solar energy coming from solar panel. The temperature and humidity sensors are used to sway the temperature and humidity of the surrounding environment. The LED light indicates the consumption of current and voltage. The consumption of current and voltage, temperature and humidity can be accessed through the Blynk App. 


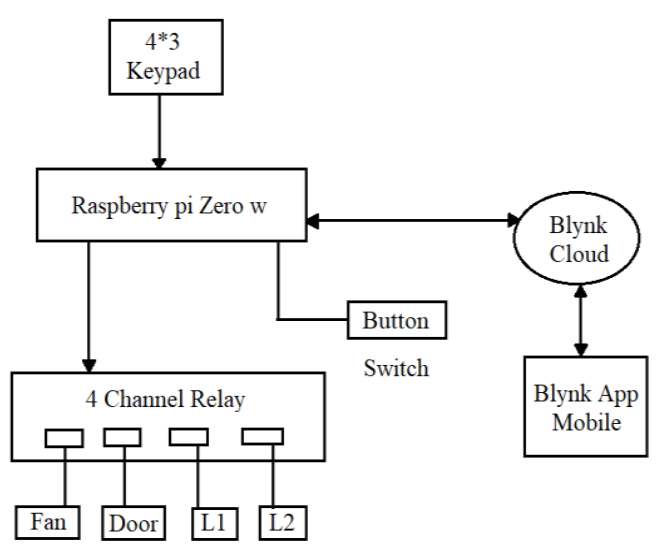

3: System Architecture of Home Automation System

Fig

\section{SECURITY FEATURE (Door Locking System):}

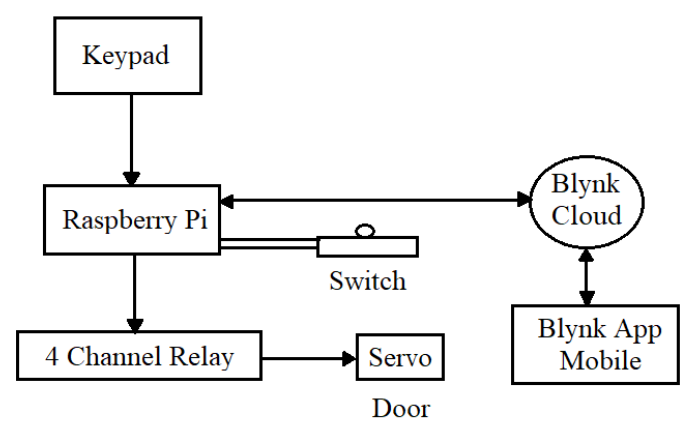

Fig 4: System Architecture of Door locking System

For security feature we are making use of Raspberry Pi. Instead of typing a fixed password we want to send the random number (4 digits) as an OTP to the registered mobile number. There would be a mobile type keypad (membrane $4 * 3$ matrix keypad) in which if user presses the button it would send an OTP to the registered mobile number. Now when user receives the OTP he/she will type it on keypad and when it is verified it will switch on the door for 15 seconds, after 15 seconds the door automatically gets closed. For door operation we have used Twilio to send and receive text messages and perform other communication function. Also this feature can be monitored in the Blynk App. Using its web services API's and servo is connected in 4 channel relay and all the operations like switching on/off light, fan etc., can be accessed through Blynk cloud.

So these are the steps to supervise the electrical appliances through Blynk App. By this procedure used we can control and monitor electrical appliances and home condition via smart phone

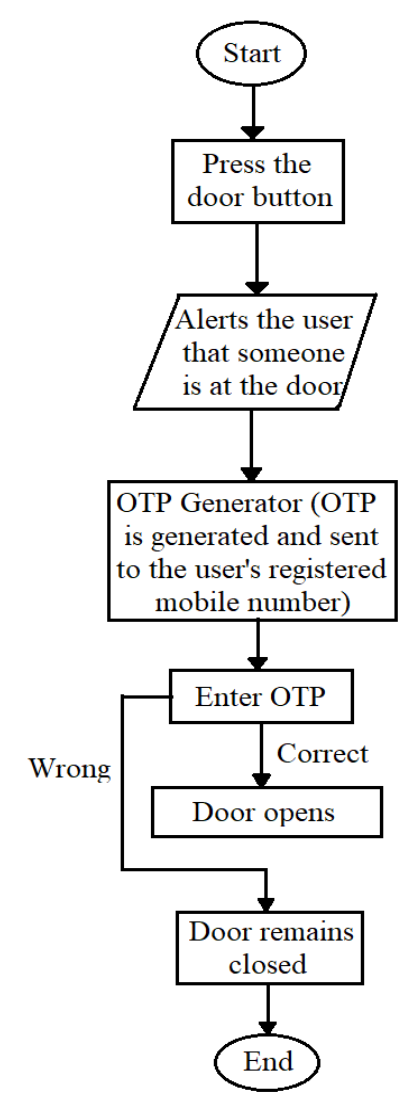

Fig 5: Flowchart of Door Locking System

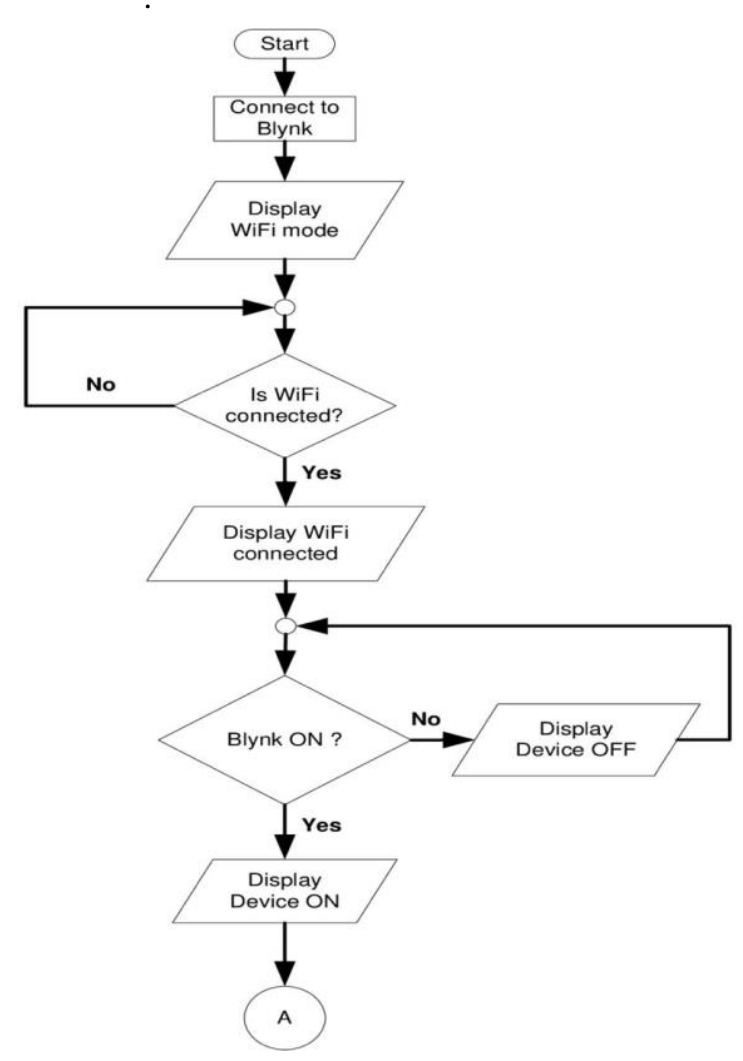

Fig 6: Control flow diagram for Blynk App 
International Journal of Engineering Applied Sciences and Technology, 2020

Vol. 5, Issue 4, ISSN No. 2455-2143, Pages 657-662

Published Online August 2020 in IJEAST (http://www.ijeast.com)

\section{EXPERIMENTAL RESULTS}

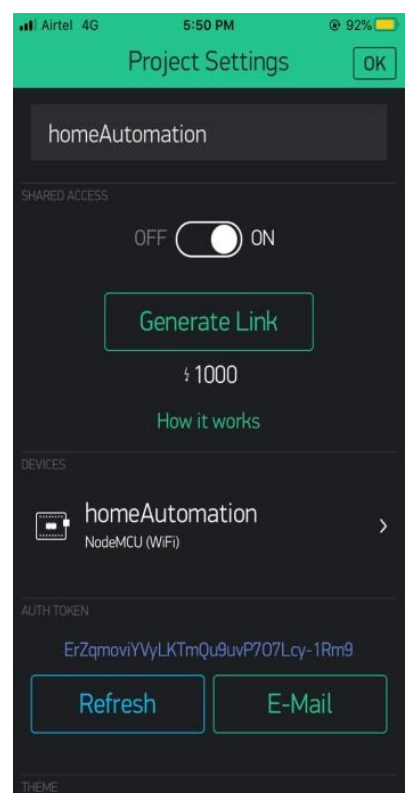

a

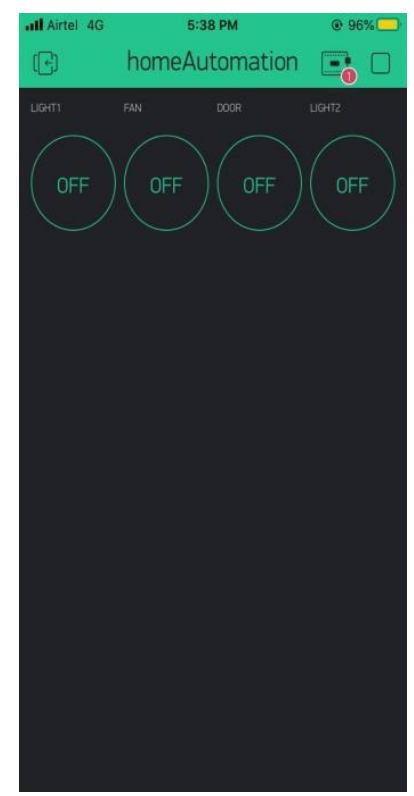

b

Fig 7: (a) Snap of blynk app indicating the token authentication to email-id. (b) Snap showing the home appliances connected and being switched off.

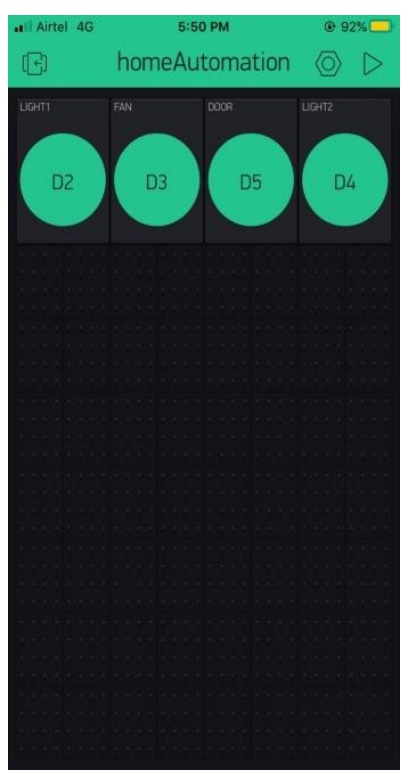

c

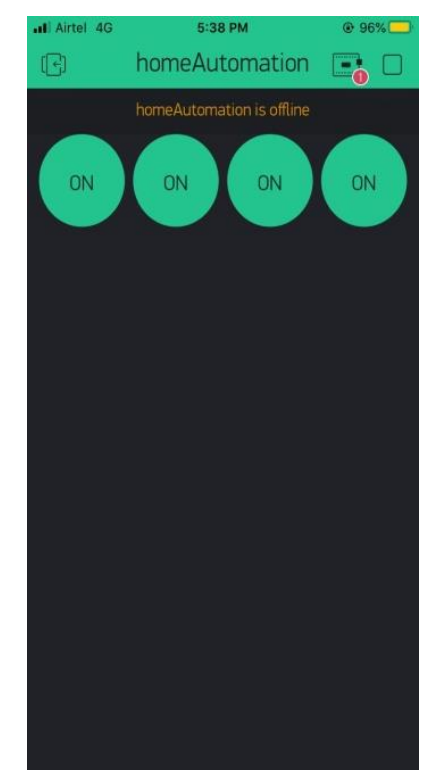

d

Fig 8: (c) Snap of pins being connected to the Node MCU. (d) Snap indicating the home appliances being switched off.

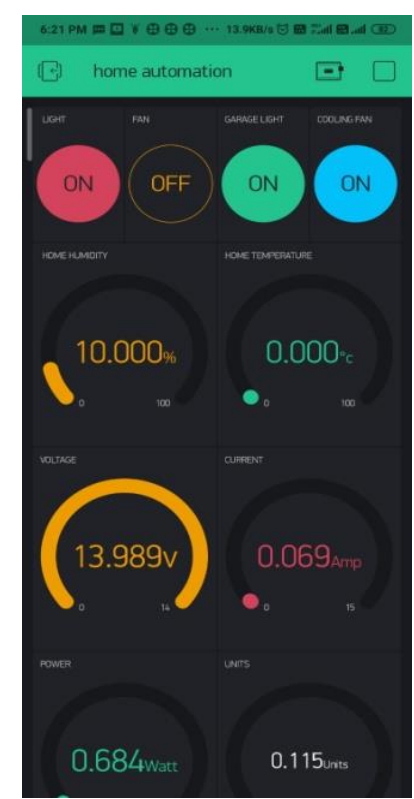

e

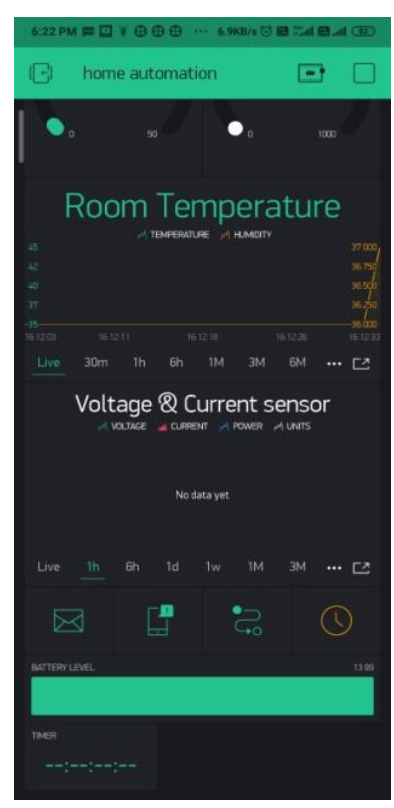

f
Fig 9: (e) Snap showing the readings of current, voltage, temperature, humidity and power consumption units. (f) Graph indicating the sensor values for a particular time period.

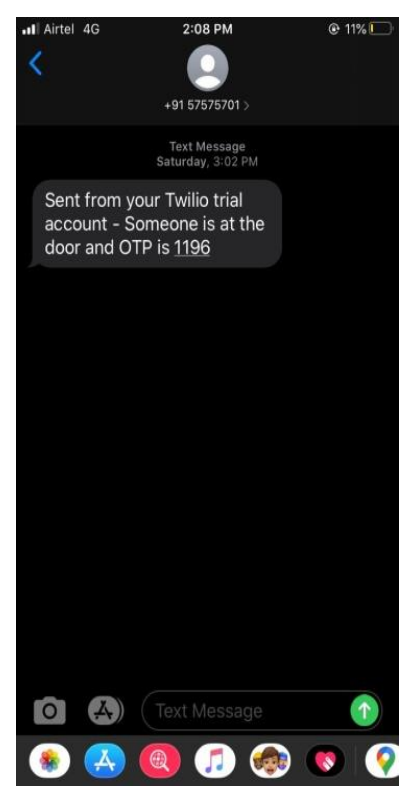

g

Fig 10: (g) Snap showing the OTP received through the text message from the Twilio trial account. 


\section{International Journal of Engineering Applied Sciences and Technology, 2020 \\ Vol. 5, Issue 4, ISSN No. 2455-2143, Pages 657-662 \\ Published Online August 2020 in IJEAST (http://www.ijeast.com)}

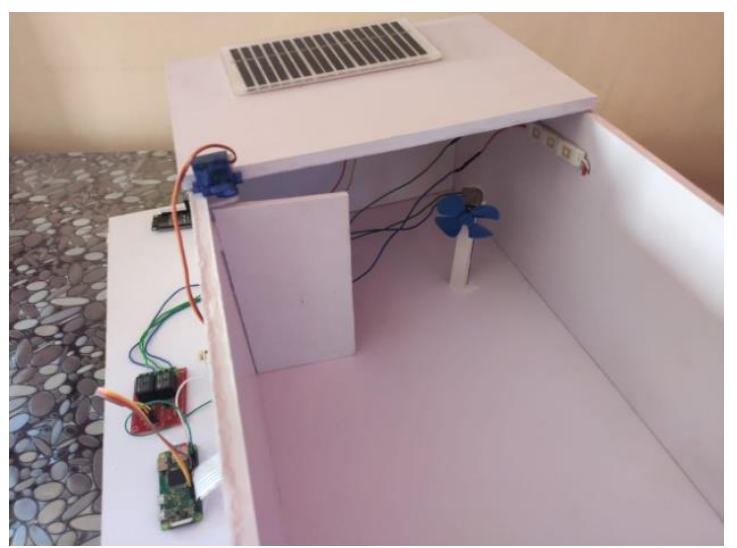

Fig 11: Solar monitoring system

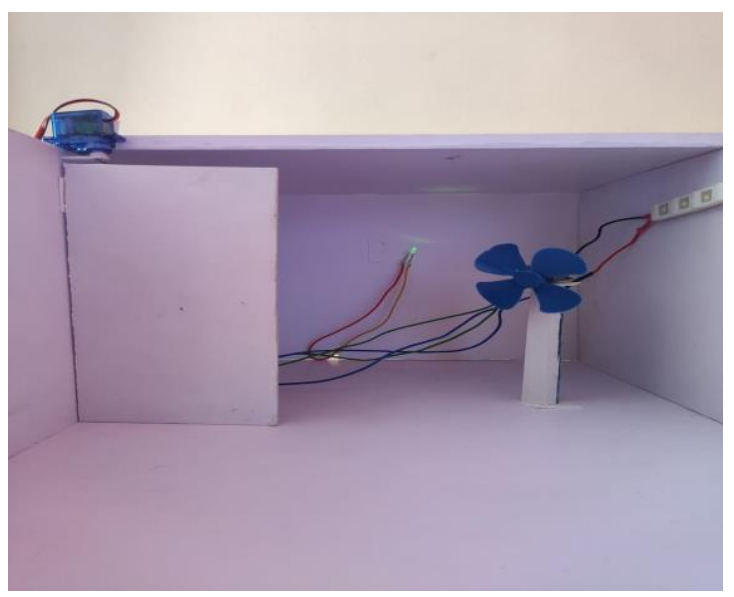

Fig 12: Snap of lights being switched on and fan

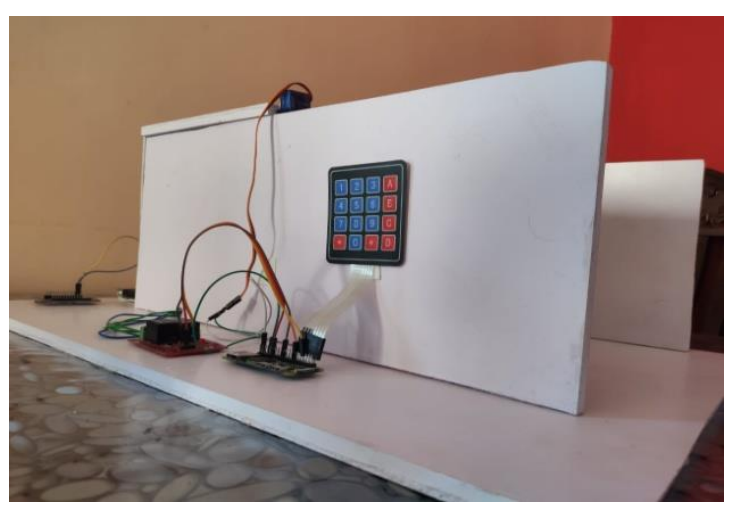

Fig 13: Door locking system using OTP

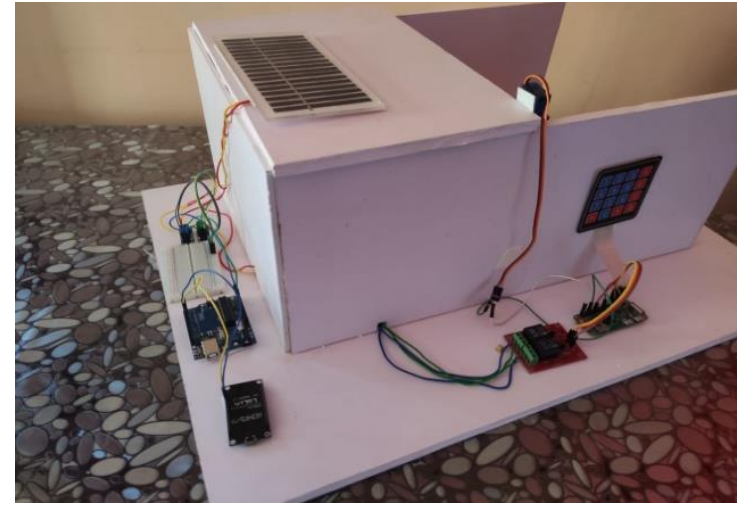

Fig 14: Snap showing components being connected

\section{CONCLUSION}

The smart home system has been proved that it works adequately by connecting the simple home appliances to it and the home devices were fruitfully authorized from a cellular mobile device. We have learnt many skills such as building the circuits and dealing with different tools that we use for this project and were able to work simultaneously as a team during this period. By dematerializing the system, we actually can provide flexibility, security and manageability to the system which we have built. By using smart portable phones and tablets we can provide cost effective system. Thus a low budget smart home system was fruitfully planned, executed and well-built.

\section{FUTURE WORK}

Future home has become a space for the tech savant with the contrivance of lots of automation technologies promoting IOT. Smart home is an actuality; a person can execute multiple chores with just one command of oral instructions. There are several new technologies which can become a part of home in near future namely, integration of smart devices, smart spaces outside home and development of smart appliances.

\section{ACKNOWLEDGEMENT}

We are actually thankful to many groups of people of who have helped us with various features of this study. We wish to thank our guide Prof. Ramya S as well as special thanks for our head of the department Dr. Sharath Kumar Y H for giving us the opportunity to do this project on "Smart Intelligence for Efficient Home Automation System".

\section{REFERENCES}

[1] Yoonil Lee, Jean King, Gabriel Underwood, Austin Sanders and Mat Osborne, 2017, "Smart PowerStrip: Home Automation by Bringing Outlets into the IoT”, 10.1109/UEMCON.2017.8249007.

[2] Waheb A. Jabbar, Mohammed Hayyan 
Alsibai, Nur Syaira S. Amran, and Samiah K. Mahayadin, 2018, "Design and Implementation of IOT-Based Automation System for Smart Home", 10.1109/ISNCC.2018.8531006.

[3] Satyendra K. Vishwakarma, Prashant Upadhyaya, Babita Kumari and Arun Kumar Mishra, 2019, "Smart Energy Efficient Home Automation system Using IoT”, 10.1109/IoT-SIU.2019.8777607.

[4] Shradha Somani, Parikshit Solunke, Shaunak oke and Parth Medhi,2019, "IoT Based Smart security and Home Automation", 10.1109/ICCUBEA.2018.8697610.

[5] J Jaya Padmini, A. Anie Selva Jothi and Ramya Harikrishna, 2019, "Advancement of Home Appliances for Home Automation Using Human Detection", 10.1109/ICECA.2019.8822140.

[6] Vikram. N, Harish K.S, Nihaal M.S, Raksha Umesh and Shetty Aashik Ashok Kumar, 2017, “A Low Cost Home Automation System Using Wi-Fi based Wireless Sensor Network Incorporating Internet of Things", 10.1109/IACC.2017.004 